\title{
Extreme Ultrafast Dynamics of Quasiparticles Excited in Surface Electronic Bands
}

\author{
P. Lazić, ${ }^{1,2}$ V. M. Silkin, ${ }^{1}$ E. V. Chulkov, ${ }^{1}$ P. M. Echenique, ${ }^{1}$ and B. Gumhalter ${ }^{1,3}$ \\ ${ }^{1}$ Donostia International Physics Center, E-20018 San Sebastian, Spain \\ ${ }^{2}$ Rudjer Bošković Institute, HR-10000 Zagreb, Croatia \\ ${ }^{3}$ Institute of Physics, HR-10000 Zagreb, Croatia \\ (Received 16 June 2006; published 25 August 2006)
}

\begin{abstract}
We develop a many-body description of the nonadiabatic dynamics of quasiparticles in surface bands valid on an extremely ultrashort time scale by combining the formalism for the calculation of quasiparticle survival probabilities with the self-consistent treatment of the electronic response of the system. Applying this approach to the benchmark $\mathrm{Cu}(111)$ surface, we assess the behavior and intervals of preasymptotic electron and hole dynamics in surface bands and locate the transition to the asymptotic regime of the exponential quasiparticle decay characterized by the corrected Fermi golden rule-type of transition rate. The general validity of these findings enables distinguishing the various regimes of ultrafast electron dynamics that may be revealed in time resolved experiments.
\end{abstract}

DOI: 10.1103/PhysRevLett.97.086801

The development of time resolved electron spectroscopies has enabled measurements of the surface electronic processes in the real time domain [1-5]. Such experiments provide direct insight into the temporal evolution of the studied systems from which information on the various relaxation processes that govern the dynamics of excited quasiparticles can be deduced. Experiments in which the time scales of relaxation are much shorter than the duration of measurement provide information on the asymptotic steady state dynamics of the excited system in its passage towards thermodynamic equilibrium. Descriptions of these processes are commonly given in terms of the rate constants that characterize asymptotic relaxation of quasiparticle states.

Methods for probing the dynamics of electronic states in confined systems are usually based on electron excitation from or injection into the system, either in the one-step processes, as in direct photoemission (PE) or inverse photoemission (IPE) spectroscopy, or in the two-step laser pump-probe induced transitions, as in two-photon photoemission (2PPE) experiments. Common to all these experiments is a sudden promotion of electrons (holes) in the initially unoccupied (occupied) states after which their motion is subjected to final state (PE and IPE) or intermediate state interactions (2PPE) with the remainder of the system. These interactions give rise to relaxation and decoherence of the excited quasiparticles [6] and produce effects in the measured spectra from which the dynamics of the excited system is assessed. Energies and lifetimes of quasiparticles in surface bands have been experimentally determined dominantly in the regimes of separated time scales of relaxation and measurement and interpreted in the framework of asymptotic decay of excited states [5].

However, if the act of measurement proceeds on the time scale comparable to or shorter than that of relaxation and decoherence processes, as in recent employments of laser pulse spectroscopies [7] or novel applications of x-ray techniques [8] with extreme temporal precision, the thus
PACS numbers: 73.20. $-\mathrm{r}, 71.10 .-\mathrm{w}, 78.47 .+\mathrm{p}, 79.60 .-\mathrm{i}$

probed quasiparticle evolution may considerably differ from asymptotic behavior described by the rate constants. Hence, reliable interpretations of measurements in this regime should be based on nonasymptotic descriptions of the evolution of excited systems. In this context, it is of particular interest to pinpoint the intervals in which descriptions of ultrafast quasiparticle dynamics in terms of few rate constants cease to be valid.

In this work, we develop the first nonasymptotic manybody description of propagation of electrons (holes) after their promotion into states within unoccupied (occupied) quasi-two-dimensional (Q2D) bands. Examples of these states are image potential, surface, or quantum-well states [3-5]. To assess ultrafast dynamics of promoted quasiparticles, we calculate the survival probability $L_{\mathbf{K}, n}(t)$ that describes quasiparticle evolution upon its injection into a 2D momentum eigenstate $|\mathbf{K}, n\rangle$ within the $n$th surface band in which its subsequent motion is affected by dynamic interactions with the substrate. These interactions give rise to intra- and interband transitions of the quasiparticle in which the energy $\omega$ and 2D momentum $\mathbf{Q}(\hbar=$ 1) are exchanged with the substrate excitations. For electron (hole) promotion into an empty (occupied) band, the survival probability is obtained as

$$
L_{\mathbf{K}, n}(t)=|G(\mathbf{K}, n, t)|^{2},
$$

where $G(\mathbf{K}, n, t)$ is the diagonal single electron (hole) propagator or Green's function giving the probability amplitude that a quasiparticle prepared at $t=0$ in the state $|\mathbf{K}, n\rangle$ and subjected to a perturbation arising from the coupling to charge density excitations in the system be recovered in the same state at $t>0$. The thus obtained quasiparticle Green's functions are prerequisites for the calculations of PE, IPE, and 2PPE spectra and rates [913]. In the case of electron injection into an empty band, $G(\mathbf{K}, n, t)$ can be expressed as [14-16]:

$$
G(\mathbf{K}, n, t)=G_{0}(\mathbf{K}, n, t) \exp [C(\mathbf{K}, n, t)]
$$


where $G_{0}(\mathbf{K}, n, t)=-i \exp \left(-i \epsilon_{\mathbf{K}, n} t\right) \theta(t)$ is the unperturbed propagator of a particle of effective mass $m_{n}$, charge $e$, and band energy $\epsilon_{\mathbf{K}, n}=E_{n}+\mathbf{K}^{2} / 2 m_{n}$, where $E_{n}$ denotes the energy of the bottom of the $n$th surface band. $C(\mathbf{K}, n, t)$ is the sum of all even order cumulants generated by the particle coupling to linear electronic response of the system [16]. For the coupling strengths typical of quasiparticles in Q2D electronic bands on metal surfaces, $C(\mathbf{K}, n, t)$ is represented with high accuracy by the second order cumulant [16] whose real and imaginary parts at zero substrate temperature assume the form:

$$
\begin{aligned}
\operatorname{Re} C_{2}(\mathbf{K}, n, t)= & -\sum_{\mathbf{Q}, n^{\prime}}\left|V_{\mathbf{Q}}\right|^{2} \int_{0}^{\infty} d \omega \mathcal{S}_{n^{\prime}, n}(\mathbf{Q}, \omega) \\
& \times\left(\frac{1-\cos \left[\left(\epsilon_{\mathbf{K}, n}-\epsilon_{\mathbf{K}+\mathbf{Q}, n^{\prime}}-\omega\right) t\right]}{\left(\epsilon_{\mathbf{K}, n}-\epsilon_{\mathbf{K}+\mathbf{Q}, n^{\prime}}-\omega\right)^{2}}\right), \\
\operatorname{Im} C_{2}(\mathbf{K}, n, t)= & -\sum_{\mathbf{Q}, n^{\prime}}\left|V_{\mathbf{Q}}\right|^{2} \int_{0}^{\infty} d \omega \frac{\mathcal{S}_{n^{\prime}, n}(\mathbf{Q}, \omega)}{\epsilon_{\mathbf{K}, n}-\epsilon_{\mathbf{K}+\mathbf{Q}, n^{\prime}}-\omega} \\
& \times\left(t-\frac{\sin \left[\left(\epsilon_{\mathbf{K}, n}-\epsilon_{\mathbf{K}+\mathbf{Q}, n^{\prime}}-\omega\right) t\right]}{\epsilon_{\mathbf{K}, n}-\epsilon_{\mathbf{K}+\mathbf{Q}, n^{\prime}}-\omega}\right) .
\end{aligned}
$$

The index $n^{\prime}$ denotes unoccupied parts of the bands allowed in intra- and interband electron transitions so that $\epsilon_{\mathbf{K}+\mathbf{Q}, n^{\prime}} \geq E_{F}, V_{\mathbf{Q}}=2 \pi e / Q$, and the spectrum of bandprojected substrate electronic excitations is given by

$$
\begin{aligned}
\mathcal{S}_{n^{\prime}, n}(\mathbf{Q}, \omega)= & \int d z_{1} \int d z_{2} f_{n^{\prime}, n}\left(z_{1}, Q\right) \\
& \times\left[-\frac{1}{\pi} \operatorname{Im} \chi\left(z_{1}, z_{2}, \mathbf{Q}, \omega\right)\right] f_{n, n^{\prime}}\left(z_{2}, Q\right) .
\end{aligned}
$$

Here $\chi\left(z_{1}, z_{2}, \mathbf{Q}, \omega\right)$ is the standard electron density response function for a system translationally invariant along the surface [5], and the oscillator strengths

$$
f_{n^{\prime}, n}\left(Q, z_{1}\right)=\int d z \phi_{n^{\prime}}(z) e^{-Q\left|z-z_{1}\right|} \phi_{n}(z)
$$

are calculated with the normal-to-the-surface component $\phi_{n}(z)$ of the $n$th band electron wave functions. Reformulation of Eqs. (3) and (4) to describe hole dynamics in occupied Q2D surface bands is straightforward [16].

Quite generally, $\operatorname{Re} C_{2}(\mathbf{K}, n, t)$ describes the decay and $\operatorname{Im} C_{2}(\mathbf{K}, n, t)$ the energy relaxation and dephasing of the initial quasiparticle state $|\mathbf{K}, n\rangle$. The evolution of $\operatorname{Re} C_{2}(\mathbf{K}, n, t)$ in the ultrashort early interval set by the Heisenberg uncertainty follows the universal "Zeno behavior" $-t^{2} / \tau_{Z}^{2}+\mathcal{O}\left(t^{4}\right)$ [17], which gives rise to Gaussian initial decoherence of $L_{\mathbf{K}, n}(t)$. In the same temporal interval, the energy relaxation and dephasing processes described by Eq. (4) cancel out each other, so as that $\operatorname{Im} C_{2}(\mathbf{K}, n, t \rightarrow 0)=\mathcal{O}\left(t^{3}\right)$. By contrast, in the opposite limit of long times, several scenarios concerning the temporal dependence of $C_{2}(\mathbf{K}, n, t)$ are possible, depending on the variation of the excitation spectrum $\mathcal{S}_{n^{\prime}, n}(\mathbf{Q}, \omega)$ across the resonant limit $\epsilon_{\mathbf{K}, n}=\epsilon_{\mathbf{K}+\mathbf{Q}, n^{\prime}}+\omega$ of the timedependent factor in the integrands in Eqs. (3) and (4). A nonvanishing and smoothly varying $\mathcal{S}_{n^{\prime}, n}(\mathbf{Q}, \omega)$ across the resonant limit gives rise to asymptotic behavior

$$
C_{2}(\mathbf{K}, n, t \rightarrow \infty) \rightarrow-\left(\Gamma_{\mathbf{K}, n} / 2+i \Lambda_{\mathbf{K}, n}\right) t-w_{\mathbf{K}, n} .
$$

Here the asymptotic decay rate or inverse lifetime

$$
\Gamma_{\mathbf{K}, n}=2 \pi \sum_{\mathbf{Q}, n^{\prime}}\left|V_{\mathbf{Q}}\right|^{2} \int_{0}^{\infty} d \omega \mathcal{S}_{n^{\prime}, n}(\mathbf{Q}, \omega) \delta\left(\epsilon_{\mathbf{K}, n}-\epsilon_{\mathbf{K}+\mathbf{Q}, n^{\prime}}-\omega\right)>0,
$$

which has the appearance of Fermi's golden rule (FGR), arises from the adiabatic limit $\pi \delta\left(\epsilon_{\mathbf{K}, n}-\epsilon_{\mathbf{K}+\mathbf{Q}, n^{\prime}}-\omega\right) t$ of the time-dependent factor in the integrand on the righthand side (RHS) of Eq. (3). The energy shift $\Lambda_{\mathbf{K}, n}$, which arises from the linear in $t$ component in the integrand on the RHS of (4), has the appearance of a RayleighSchrödinger correction to unperturbed energy and can be absorbed in the renormalization of $E_{n}$ since it builds up on the extremely short time scale $\tau_{s} \sim 0.2$ fs $[18,19]$. The time-independent term $w_{\mathbf{K}, n}$ is the off-resonant correction to the first two terms on the RHS of (7) and measures the nonadiabaticity of excitation processes following the sudden switching on of the quasiparticle interaction with the substrate response. In the opposite situation of $\mathcal{S}_{n^{\prime}, n}(\mathbf{Q}, \omega)$ varying discontinuously across the resonant limit, the asymptotic form of $C_{2}(\mathbf{K}, n, t)$ exhibits a more complicated behavior leading to a nonexponential decay of the quasiparticle. Hence, further insight in the quasiparticle dynamics on both the ultrashort (preasymptotic) and long (asymptotic) time scales requires specification of the excitation spectrum (5).

In this Letter, we illustrate different stages of ultrafast quasiparticle dynamics in surface bands for the benchmark surface $\mathrm{Cu}(111)$ with one electron introduced in the first image state (IS) band or one hole created in the surface state (SS) band, for which the asymptotic excited state energies and lifetimes are available from experimental and theoretical studies [1-5]. We start from the selfconsistent RPA response function $\chi\left(z_{1}, z_{2}, \mathbf{Q}, \omega\right)$ calculated for a $N$-layer slab $(N=31)$ that models the $\mathrm{Cu}(111)$ surface by using the effective potential [20] and the method described in Ref. [21]. This model enables a systematic treatment of intra- and interband transitions on equivalent footing. The oscillator strengths (6) were calculated using linear combinations of pairs of symmetric and antisymmetric electron wave functions from the same slab calculation. From this, we computed the excitation spectrum (5) on a $(Q, \omega)$ grid for combinations of indices 
$\left(0 \leq n, n^{\prime} \leq n_{\max }\right)$ that cover the relevant intra- and interband transitions. Details of this calculation and the results for electron dynamics on other surfaces will be presented elsewhere.

Figure 1 shows the computed intensity of the ISprojected intraband component $\left(n^{\prime}=n=\mathrm{IS}\right)$ of the excitation spectrum $\mathcal{S}_{n^{\prime}, n}(\mathbf{Q}, \omega)$ over the phase space of excitation energies $\omega$ and wave vectors $Q$ relevant in the calculations of $L_{\mathbf{K}, n}(t)$. Dominant contributions to the intensity come from the surface plasmon which disperses along a parabolic curve starting at the point $Q=0$ and $\omega=7.6 \mathrm{eV}$, the bulk plasmon whose dispersion curve starts at $\omega=12.6 \mathrm{eV}$, and the electron-hole $(e-h)$ quasicontinuum with maximum intensity in the region encompassed by the parabolas $\omega=Q^{2} / 2 m_{n} \pm Q v_{F, n}$ and $0 \leq \omega \leq Q v_{F, n}-Q^{2} / 2 m_{n}\left(v_{F, n}\right.$ is the Fermi velocity in the $n$th band). Qualitatively similar behavior of (5) is obtained for other combinations of indices $n$ and $n^{\prime}$.

The quasiparticle survival probability (1) is determined by the behavior of $\operatorname{Re} C_{2}(\mathbf{K}, n, t)$ given by Eq. (3). It depends on the $(\mathbf{Q}, \omega)$ integral over the product of a time-independent factor $\mathcal{W}_{n^{\prime}, n}(\mathbf{K}, \mathbf{Q}, \omega)=$ $Q /(2 \pi)^{2}\left|V_{\mathbf{Q}}\right|^{2} \mathcal{S}_{n^{\prime}, n}(\mathbf{Q}, \omega) /\left(\epsilon_{\mathbf{K}, n}-\epsilon_{\mathbf{K}+\mathbf{Q}, n^{\prime}}-\omega\right)^{2}$, which becomes singular at $\epsilon_{\mathbf{K}, n}-\epsilon_{\mathbf{K}+\mathbf{Q}, n^{\prime}}=\omega$, and a bounded time-dependent factor $\left(1-\cos \left[\left(\epsilon_{\mathbf{K}, n}-\epsilon_{\mathbf{K}+\mathbf{Q}, n^{\prime}}-\omega\right) t\right]\right)$, which may quench this singularity. In Fig. 2 , we show the variation of $\mathcal{W}_{n^{\prime}, n}(\mathbf{K}, \mathbf{Q}, \omega)$ over the $(Q, \omega)$ plane for $n^{\prime}=n=$ IS and the initial electron state at the IS-band bottom $K=0$. The region around the integrable singularity at $(Q=0, \omega=0)$ gives the dominant contribution to $\operatorname{Re} C_{2}(\mathbf{K}=0$, IS, $t)$ in the femtosecond domain and, thereby, to decoherence of the quasiparticle. However, it does not contribute to its exponential decay, because for

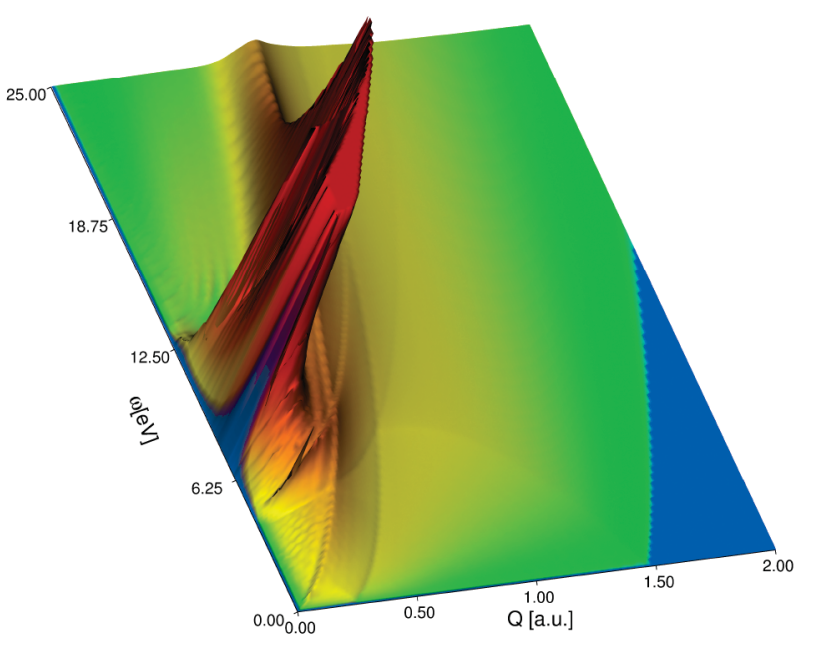

FIG. 1 (color). Intraband image potential state-projected component $\left(n^{\prime}=n=\mathrm{IS}\right)$ of the slab electronic density excitation spectrum $\mathcal{S}_{n^{\prime}, n}(\mathbf{Q}, \omega)$ [Eq. (5)] shown as a function of energy $\omega$ and momentum $Q$ of the excitations.
$K=0$ only the interband transitions $n^{\prime}<$ IS can give a contribution to $\Gamma_{\mathbf{K} \text {,IS }}$, as is evident from the argument of the $\delta$ function on the RHS of Eq. (8). For $K>0$, the singularity moves away and its weight is redistributed over a larger segment of the $(Q, \omega)$-phase space [22]. This effective increase of the phase space for quasiparticle scattering by substrate excitations gives rise to the onset of intraband contributions and, thereby, to enhancement of the total decay rate $\Gamma_{\mathbf{K}, \text { IS }}$. On the other hand, the largest phase space for intraband hole decay is for initial states at the band bottom $(K=0)$ because, in inelastic transitions, the holes decay only towards the Fermi level.

Temporal behavior of the survival probability (1) can now be calculated for initial values of $n$ and $\mathbf{K}$ using the above obtained results. Figure 3 shows $L_{\mathbf{K}, \text { IS }}(t)$ for an electron created in the IS band on $\mathrm{Cu}(111)$ surface with initial wave vectors $K=0.01$ and $K=0.05$ a.u. The very early Gaussian decay is superseded by a superposition of oscillations arising from nonadiabatic (off-resonant) excitations of surface plasmons in the slab and a gradual buildup of the $w_{\mathbf{K}, n}$-corrected FGR decay (7) arising from resonant excitation of $e-h$ pairs. Because of the offthe-energy-shell character of plasmon excitations, their amplitude diminishes as $t \rightarrow \infty$. We find that for $K=$ 0.01 interband IS $\rightarrow$ SS transitions contribute about $39 \%$ to the total decay rate $\Gamma_{\mathbf{K}, I S}$, and the remaining $61 \%$ arise from interband transitions into bulk bands, in agreement with earlier calculations [5]. The contribution from intraband IS $\rightarrow$ IS electron transitions to the resonant decay rate $\Gamma_{\mathbf{K}, \text { IS }}$ is insignificant at this value of $K$, as only very small initial kinetic energy is available for excitation of other electrons in the system. On the other hand, their role is dominant in the off-resonant excitation of surface plas-

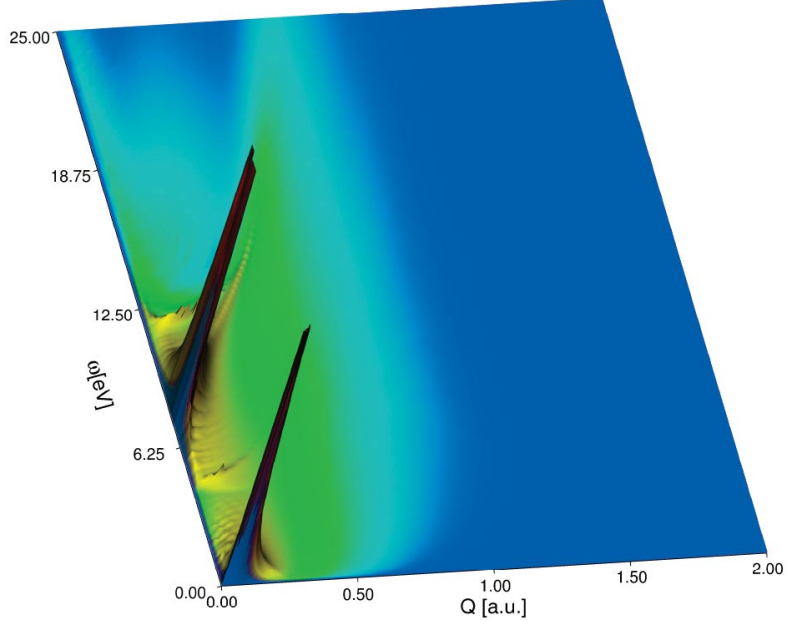

FIG. 2 (color). Intensity plot of the unbounded component $W_{n^{\prime}, n}(\mathbf{K}, \mathbf{Q}, \omega)$ of the integrand in (3) showing the regions in $(Q, \omega)$-phase space of substrate excitations that give major contributions to intraband-induced decoherence of an image potential state electron $\left(n=n^{\prime}=\mathrm{IS}\right)$ with initial $K=0$. 


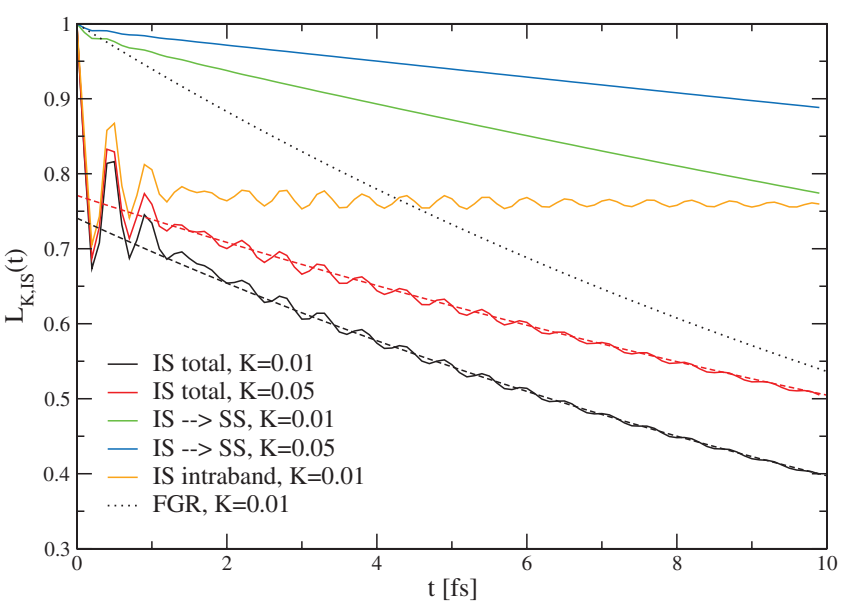

FIG. 3 (color). Intra- and interband components and total survival probability $L_{\mathbf{K}, I S}(t)$ for an electron promoted into the first image state band on $\mathrm{Cu}(111)$ with initial state wave vector $K=0.01$ and $K=0.05$ a.u. (solid curves, see legend for assignments). Asymptotic decay given by "corrected" FGR law [Eq. (7)] is shown by dashed curves and "bare" FGR exponential decay for $K=0.01$ a.u. by a dotted curve.

mons and the buildup of nonadiabatic correction $w_{\mathbf{K}, \text { IS }}=$ 0.15 . Qualitatively similar trends are retained for initial electron wave vector $K=0.05$ a.u.

Figure 4 shows various contributions to the survival probability (1) for a hole created in the SS band on $\mathrm{Cu}(111)$ with initial $K=0.01$ and $K=0.05$ a.u. Here, in contrast to the case of IS-electron decay, the intraband $\mathrm{SS} \rightarrow \mathrm{SS}$ transitions for $K=0.01$ a.u. contribute the major part $(\sim 70 \%)$ to the total decay rate $\Gamma_{\mathbf{K}, \mathrm{SS}}$, again in accord with earlier calculations [5]. They also give a dominant contribution $(\sim 60 \%)$ to the short time behavior of $L_{\mathbf{K}, \mathrm{SS}}(t)$ reflected in the magnitude of the total non-

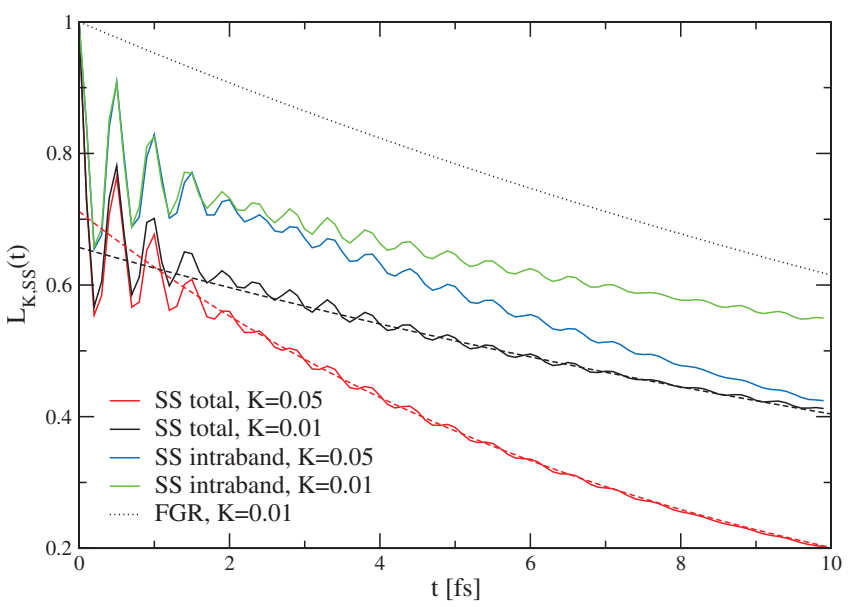

FIG. 4 (color). Same as in Fig. 3 for the survival probability $L_{\mathbf{K}, \mathrm{SS}}(t)$ of a hole created in a $\mathrm{Cu}(111)$ surface state band. adiabatic correction $w_{\mathbf{K}, \mathrm{SS}}=0.21$. A similar situation holds for $K=0.05$ a.u.

In conclusion, the results of calculations of quasiparticle survival probabilities shown in Figs. 3 and 4 enable the identification of three distinct regimes of ultrafast quasiparticle dynamics in IS and SS bands on $\mathrm{Cu}(111)$. The early Zeno regime $(0<t<1 \mathrm{fs})$ is followed by preasymptotic non-Markovian evolution with superimposed offresonant excitation of surface plasmons and resonant excitation of $e-h$ pairs. This structure persists up to $t \sim 10 \mathrm{fs}$, and only past that time do the off-resonant plasmon excitations die out, and the steady state asymptotic evolution governed by the corrected FGR decay (7) takes over. However, even long past that time, the bare FGR decay $\exp \left(-\Gamma_{\mathbf{K}, n} t\right)$ is not yet approached, signifying that extreme ultrafast dynamics of quasiparticles promoted in surface bands requires preasymptotic description of relaxation and decay processes. Hence, further progress in theoretical interpretations of the corresponding PE, IPE, and 2PPE spectra should be sought in terms of the above elucidated quasiparticle evolution rather than its approximations based on asymptotic rate constants.

[1] T. Hertel et al., Phys. Rev. Lett. 76, 535 (1996); M. Wolf, E. Knoesel, and T. Hertel, Phys. Rev. B 54, R5295 (1996).

[2] H. Petek and S. Ogawa, Prog. Surf. Sci. 56, 239 (1997).

[3] U. Höfer et al., Science 277, 1480 (1997).

[4] M. Weinelt, J. Phys. Condens. Matter 14, R1099 (2002).

[5] P. M. Echenique et al., Surf. Sci. Rep. 52, 219 (2004).

[6] F. El-Shaer and B. Gumhalter, Phys. Rev. Lett. 93, 236804 (2004).

[7] A. Kubo et al., Nano Lett. 5, 1123 (2005).

[8] A. Föhlisch et al., Nature (London) 436, 373 (2005).

[9] C. Caroli et al., Phys. Rev. B 8, 4552 (1973).

[10] J. B. Pendry, J. Phys. C 14, 1381 (1981).

[11] H. Ueba, Surf. Sci. 334, L719 (1995).

[12] M. Sakaue et al., Phys. Rev. B 66, 094302 (2002).

[13] C. Timm and K. H. Bennemann, J. Phys. Condens. Matter 16, 661 (2004).

[14] D. Dunn, Can. J. Phys. 53, 321 (1975).

[15] G. D. Mahan, Many Particle Physics (Plenum, New York, 1981), Chap. 6.

[16] B. Gumhalter, Phys. Rev. B 72, 165406 (2005).

[17] P. Facchi, H. Nakazato, and S. Pascazio, Phys. Rev. Lett. 86, 2699 (2001).

[18] B. Gumhalter, Surf. Sci. 518, 81 (2002).

[19] A. Borisov et al., Chem. Phys. Lett. 387, 95 (2004).

[20] E. V. Chulkov, V. M. Silkin, and P. M. Echenique, Surf. Sci. 437, 330 (1999).

[21] V. M. Silkin et al., Phys. Rev. B 72, 115435 (2005).

[22] See EPAPS Document No. E-PRLTAO-97-049635 for an animated illustration of the redistribution of the quantity shown in Fig. 2 with the variation of the IS-electron incident wave vector $K$ in the interval $0<K<1.9$ a.u. For more information on EPAPS, see http://www.aip.org/ pubservers/epaps.html. 